\title{
Effect of Dietary Cabbage Fermentation Extract and Young Barley Leaf Powder on Immune Function of Sprague-Dawley Rats
}

\author{
Yoshiyuki MiYazaki, Yoko ToKunaga, Kinya TAKAgAKI ${ }^{1}$, Sinji TsusakI ${ }^{1}$, \\ Hirofumi TACHIBANA and Koji Yamada* \\ Laboratory of Food Chemistry, Division of Applied Biological Chemistry, Department of \\ Bioscience and Biotechnology, Faculty of Agriculture, Kyushu University \\ 46-09, 6-10-1 Hakozaki, Higashi-ku, Fukuoka 812-8581, Japan \\ ${ }^{1}$ Department of Research and Development, Toyo Shinyaku Co. Ltd., \\ 2-19-27, Hakataekimae, Hakata-ku, Fukuoka, 812-0011, Japan
}

(Received October 12, 2000)

\begin{abstract}
Summary We investigated dietary effects of cabbage fermentation extract (CFE) and young barley leaf powder (YBLP) on rat immune functions. Male Sprague-Dawley rats of $4 \mathrm{wk}$ age were fed for $3 \mathrm{wk}$ diets containing these samples at 0.1 or $1 \%$ level. After the feeding period, serum IgG level was significantly higher in the rats fed 1\% CFE. IgG productivity of spleen lymphocytes was enhanced dose-dependently in both groups of CFE and YBLP. Furthermore, IgG productivity of mesenteric lymph node (MLN) lymphocytes was approximately 2 times higher in the rats fed 1\% CFE diet than in the control ones. IgA productivity of MLN lymphocytes tended to increase in both of CFE and YBLP groups. From these results, it was suggested that dietary CFE and YBLP reinforced Ig productivity in both systemic and intestinal immune systems. Moreover, CFE feeding tended to enhance the production of TNF- $\alpha$ by spleen lymphocytes. In spleen phospholipids, the level of arachidonic acid, a substrate for inflammatory lipid mediators, was not affected by CFE or YBLP feeding.

Key Words cabbage fermentation extract, young barley leaf powder, serum immunoglobulin level, immunoglobulin production, rat lymphocytes
\end{abstract}

Recently, functions in addition to nutritional values and taste have been looked upon as important factors of foodstuffs, and many kinds of functional foods have been developed. For this reason, investigations on physiological effects of functional foods are required.

In previous studies, we have shown that components in plants, for example dietary fibers $(1,2)$, natural food colorings (3) and vegetable extracts (4), have regulatory effects on Immunoglobulin (Ig) production. These results suggest that foodstuffs derived from vegetables have functions useful for modulation of Ig production. Ig plays an important role in humoral immunity and consists of five classes different in molecular sizes, structures and functions. IgA prevents invasion of exogenous substances from intestinal tract, $\operatorname{IgG}$ serves as a main factor for detoxification and clearance of foreign substances in the systemic immune system, and IgM rapidly responds to primary infections. Therefore, we considered that a rise in the level and production of $\operatorname{Ig} \mathrm{A}$, $\operatorname{IgG}$ and $\operatorname{IgM}$ was connected with reinforcement of the

* To whom correspondence should be addressed.

E-mail: yamadako@agr.kyushu-u.ac.jp

Abbreviations: CFE, cabbage fermentation extract; FBS, fetal bovine serum; ELISA, enzyme-linked immunosorbent assay; Ig, immunoglobulin; MLN, mesenteric lymph node; PC, phosphatidylcholine; PE, phosphatidylethanolamine; PUFA, polyunsaturated fatty acid; TNF, tumor necrosis factor; YBLP, young barley leaf powder. immune system.

On the other hand, eicosanoids such as prostaglandin and leukotriene also play important roles in the immune system. They are biosynthesized from polyunsaturated fatty acid (PUFA) and closely involved as triggers in inflammatory response and type I allergy $(5,6)$. We have previously reported that dietary fats and some kind of fatty acids modulate production and release of eicosanoids $(7-10)$. These results suggest that lipid metabolism in the liver and the proportion of PUFA in immune-related tissues and cells are responsible for the modulation of eicosanoid production.

In this study, we investigated dietary effects of cabbage fermentation extract (CFE) and young barley leaf powder (YBLP) on the immune functions in SpragueDawley (SD) rats.

\section{MATERIALS AND METHODS}

Materials. CFE and YBLP were prepared in Toyo Shinyaku Company, Ltd. (Tokyo, Japan). CFE is a dried powder extracted from fermented chinese cabbage. Washed and milled cabbage leaves were fermented with a lactic acid bacterium, Lactobacillus plantarum. The fermented products were then filtrated and concentrated. After sterilization, the extracts were spray-dried with dextrin. YBLP is a dried powder of young barley leaves. Barley leaves grown to about $30 \mathrm{~cm}$ in height were mowed, washed, and dried. The dry leaves were powdered as such without further processing. In the prepa- 
Table 1. Effects of dietary CFE and YBLP on growth and food intake of rats.

\begin{tabular}{cccccc}
\hline & $\begin{array}{c}\text { Initial body } \\
\text { weight }(\mathrm{g})\end{array}$ & $\begin{array}{c}\text { Final body } \\
\text { weight }(\mathrm{g})\end{array}$ & $\begin{array}{c}\text { Weight gain } \\
(\mathrm{g})\end{array}$ & $\begin{array}{c}\text { Food intake } \\
(\mathrm{g} / \mathrm{d})\end{array}$ & $\begin{array}{c}\text { Food efficiency } \\
(\mathrm{g} \text { gain/g intake })\end{array}$ \\
\hline $\begin{array}{l}\text { None } \\
\text { CFE }\end{array}$ & $121 \pm 01$ & $271 \pm 07$ & $150 \pm 07^{\mathrm{ab}}$ & $16.2 \pm 0.3$ & $0.44 \pm 0.02^{\mathrm{a}}$ \\
$0.1 \%$ & $121 \pm 03$ & $277 \pm 13$ & $156 \pm 11^{\mathrm{b}}$ & $17.0 \pm 0.7$ & $0.43 \pm 0.01^{\mathrm{a}}$ \\
$1 \%$ & $121 \pm 02$ & $270 \pm 08$ & $149 \pm 08^{\mathrm{ab}}$ & $16.0 \pm 0.4$ & $0.44 \pm 0.02^{\mathrm{a}}$ \\
YBLP & & & & & \\
$0.1 \%$ & $121 \pm 04$ & $260 \pm 05$ & $139 \pm 04^{\mathrm{ab}}$ & $16.7 \pm 0.6$ & $0.40 \pm 0.02^{\mathrm{ab}}$ \\
$1 \%$ & $121 \pm 03$ & $252 \pm 08$ & $130 \pm 06^{\mathrm{a}}$ & $16.7 \pm 0.6$ & $0.37 \pm 0.02^{\mathrm{b}}$ \\
\hline
\end{tabular}

Data are means \pm SE $(n=4$ or 5$)$, and ${ }^{\mathrm{a}, \mathrm{b}}$ values not sharing a common superscript letter are significantly different at $p<0.05$.

ration of experimental diets, these samples were partially replaced with cornstarch.

Animals and diets. Four-week-old male SD rats were purchased from Seac Yoshitomi (Fukuoka, Japan) and individually housed in a room with a controlled temperature of $20-23^{\circ} \mathrm{C}$ and light cycle of $08: 00$ to $20: 00$. After acclimatizing for $5 \mathrm{~d}$, rats were divided into 5 groups of 5 rats each and allowed free access to the experimental diets and to deionized water. The diets which were prepared according to the recommendation of the American Institute of Nutrition (AIN-93G), contained $36.7 \%$ cornstarch, $20 \%$ casein, $13.2 \% \alpha$-cornstarch, $10 \%$ sucrose, $10 \%$ safflower oil, $5 \%$ cellulose, $3.5 \%$ mineral mixture, $1 \%$ vitamin mixture, $0.3 \% \mathrm{~L}-$ cystine, $0.25 \%$ choline bitartrate and $0.002 \%$ tertbutylhydroquinone. After 3 wk's feeding, the rats were killed by withdrawing blood from the abdominal aorta under diethyl ether anesthesia. The liver, heart, lung, kidney, spleen and adipose tissue of each rat were immediately excised and weighed. Lymphocytes were isolated from spleen and mesenteric lymph node (MLN) to measure their Ig productivity. This experiment was carried out in line with the Guidelines for Animal Experiment in Faculty of Agriculture and the Graduate School, Kyushu University and the law (No. 105) and notification (No. 6) of the government.

Cells and cell culture. Spleen and MLN lymphocytes were isolated from rats fed CFE or YBLP diet for $3 \mathrm{wk}$ using Lympholyte-rat (Cedarlene, Hornby, Canada). Cells were cultured for $24 \mathrm{~h}$ in RPMI 1640 medium (Nissui Pharmaceutical, Tokyo, Japan) supplemented with 10\% fetal bovine serum (FBS) (Bio Whittaker, Walkersville, MD), as described previously (11). IgA, IgG and IgM contents in the serum and culture supernatant were determined by enzyme-linked immunosorbent assay (ELISA) as described previously (11). Tumor necrosis factor alpha (TNF- $\alpha$ ) content in the culture supernatant of spleen lymphocytes was measured using an assay kit (Cosmo Bio Co. Ltd., Tokyo, Japan) according to the method of Stepaniak et al. (12).

Lipid analysis. The tissue lipids were extracted by the method of Folch et al. (13). Spleen phosphatidylcholine (PC) and phosphatidylethanolamine (PE) were separated by thin-layer chromatography (14), and the fatty acid composition was analyzed by GLC (GC-14B, Shimadzu Co., Kyoto, Japan) in a Supelcowax 10 col-
Table 2. Effects of dietary CFE and YBLP on serum immunolobulin levels.

\begin{tabular}{lccc}
\hline & $\begin{array}{c}\mathrm{IgA} \\
(\mu \mathrm{g} / \mathrm{mL})\end{array}$ & $\begin{array}{c}\mathrm{IgG} \\
(\mathrm{mg} / \mathrm{mL})\end{array}$ & $\begin{array}{c}\operatorname{IgM} \\
(\mu \mathrm{g} / \mathrm{mL})\end{array}$ \\
\hline $\begin{array}{l}\text { None } \\
\text { CFE }\end{array}$ & $24.8 \pm 1.1$ & $1.4 \pm 0.1^{\mathrm{a}}$ & $266 \pm 27^{\mathrm{a}}$ \\
$0.1 \%$ & $22.6 \pm 1.3$ & $1.3 \pm 0.1^{\mathrm{a}}$ & $187 \pm 20^{\mathrm{b}}$ \\
$1 \%$ & $23.8 \pm 2.5$ & $1.9 \pm 0.1^{\mathrm{b}}$ & $186 \pm 24^{\mathrm{b}}$ \\
YBLP & & & \\
$0.1 \%$ & $24.7 \pm 1.5$ & $1.2 \pm 0.1^{\mathrm{a}}$ & $262 \pm 20^{\mathrm{ab}}$ \\
$1 \%$ & $23.2 \pm 1.9$ & $1.5 \pm 0.1^{\mathrm{a}}$ & $243 \pm 30^{\mathrm{ab}}$ \\
\end{tabular}

Data are means \pm SE ( $n=4$ or 5 ), and ${ }^{a, b}$ values not sharing a common superscript letter are significantly different at $p<0.05$.

umn $(60 \mathrm{~m} \times 0.32 \mathrm{~mm}$; Supelco INC., Bellefonte, PA, USA) as the methyl ester. The GLC conditions were as follows: column temperature, $220^{\circ} \mathrm{C}$; injector temperature, $250^{\circ} \mathrm{C}$; detector temperature, $250^{\circ} \mathrm{C}$; flow rate of helium, $1.6 \mathrm{~mL} / \mathrm{min}$; split ratio, $1 / 20$.

Statistics. Data are expressed as the means \pm SE $(n=4$ or 5 ) and were analyzed by one-way ANOVA followed by Duncan's new multiple-range test to estimate the significance of differences among experimental groups (15).

\section{RESULTS}

\section{Effects of dietary CFE and YBLP on growth of rats}

As shown in Table 1, there was no significant difference in weight gain and food efficiency between the CFE group and the control. In the YBLP group, weight gain was suppressed dose-dependently, whereas food intake was not different from that in the control. Consequently, food efficiency was significantly decreased by YBPL feeding. The weights of white adipose tissue, kidney, liver, lung, spleen and heart were not affected by CFE or YBLP feeding (data not shown).

Effects of dietary CFE and YBLP on serum Ig levels and Ig productivity of rat lymphocytes

As shown in Table 2, CFE significantly affected serum levels of Ig except IgA. Serum IgG level in CFE-fed rats was significantly higher than that in the control. On the contrary, CFE feeding caused a decrease in serum IgM level. YBLP did not significantly affect any serum Ig 
Table 3. Effects of dietary CFE and YBLP on immunoglobulin productivity of spleen and mesenteric lymph node lymphocytes.

\begin{tabular}{|c|c|c|c|c|c|c|}
\hline & \multicolumn{6}{|c|}{ Immunoglobulin concentration (ng/mL) } \\
\hline & \multicolumn{3}{|c|}{ Spleen } & \multicolumn{3}{|c|}{ MLN } \\
\hline & $\operatorname{Ig} \mathrm{A}$ & $\operatorname{IgG}$ & $\operatorname{IgM}$ & $\operatorname{Ig} \mathrm{A}$ & $\operatorname{IgG}$ & $\operatorname{IgM}$ \\
\hline None & $16.1 \pm 2.4$ & $29.3 \pm 1.7^{\mathrm{a}}$ & $60.3 \pm 4.2^{\mathrm{a}}$ & $9.3 \pm 3.4$ & $25.6 \pm 1.7^{\mathrm{a}}$ & $12.1 \pm 1.5^{\mathrm{a}}$ \\
\hline \multicolumn{7}{|l|}{ CFE } \\
\hline $0.1 \%$ & $16.3 \pm 2.2$ & $40.2 \pm 4.7^{\mathrm{ab}}$ & $47.9 \pm 3.2^{\mathrm{b}}$ & $13.6 \pm 2.2$ & $33.6 \pm 2.1^{\mathrm{a}}$ & $8.4 \pm 1.3^{\mathrm{ab}}$ \\
\hline $1 \%$ & $17.0 \pm 2.2$ & $47.3 \pm 7.0^{\mathrm{b}}$ & $45.7 \pm 5.4^{\mathrm{b}}$ & $16.6 \pm 3.5$ & $51.2 \pm 4.5^{\mathrm{b}}$ & $7.6 \pm 1.1^{\mathrm{b}}$ \\
\hline \multicolumn{7}{|l|}{ YBLP } \\
\hline $0.1 \%$ & $19.5 \pm 2.3$ & $41.2 \pm 5.6^{\mathrm{ab}}$ & $64.3 \pm 3.5^{\mathrm{a}}$ & $10.9 \pm 0.6$ & $23.8 \pm 1.1^{\mathrm{a}}$ & $6.3 \pm 0.3^{b}$ \\
\hline $1 \%$ & $20.1 \pm 1.9$ & $43.2 \pm 4.1^{\mathrm{ab}}$ & $60.0 \pm 3.0^{\mathrm{a}}$ & $11.1 \pm 3.2$ & $25.8 \pm 4.6^{\mathrm{a}}$ & $8.0 \pm 1.9^{b}$ \\
\hline
\end{tabular}

Data are means \pm SE $(n=4$ or 5$)$, and ${ }^{\text {a,b }}$ values not sharing a common superscript letter are significantly different at $p<0.05$.

level.

Table 3 shows the Ig productivities of spleen and MLN lymphocytes in the rats fed CFE and YBLP diets. The IgA productivity was not affected, whereas the IgG productivity of spleen lymphocytes was significantly enhanced by CFE feeding. The IgM productivity of spleen lymphocytes in the rats fed CFE was significantly lower than in the control. YBLP feeding tended to enhance the IgG production by spleen lymphocytes in a dose-dependent manner. The IgA and IgM productivities of spleen lymphocytes were not affected by YBLP feeding. On the other hand, CFE feeding at a 10\% level significantly increased the IgG productivity of MLN lymphocytes. The IgA productivity tended to increase in MLN lymphocytes from the rats fed CFE and YBLP diets.

Effects of dietary CFE and YBLP on production of TNF- $\alpha$ by rat spleen lymphocytes

As shown in Fig. 1, the TNF- $\alpha$ production by spleen lymphocytes tended to be enhanced in CFE-fed groups. On the other hand, YBLP feeding did not affect the production of TNF- $\alpha$.

Effects of dietary CFE and YBLP on fatty acid composition of spleen phospholipids

After 3 wk's feeding, we measured fatty acid composition of spleen PC (Table 4). Myristate and palmitate contents were lower in the CFE- or YBLP-fed rats than in the control. CFE or YBLP feeding at a 1\% level also affected the contents of some kinds of PUFA. Arachidonic acid content was increased, and docosahexaenoic acid content was decreased. Dramatic changes of PUFA contents in spleen PC, however, were not observed. On the other hand, the composition of PUFA in PE was not affected by either CFE or YBLP feeding (data not shown).

\section{DISCUSSION}

In this study, we investigated dietary effects of two functional foods, CFE and YBLP, on immune functions of SD rats.

CFE is a dried powder extracted from cabbage leaves fermented by a sort of lactic acid bacterium. It has been long thought that components in cabbage have anticancer properties $(16,17)$. In this regard, we showed

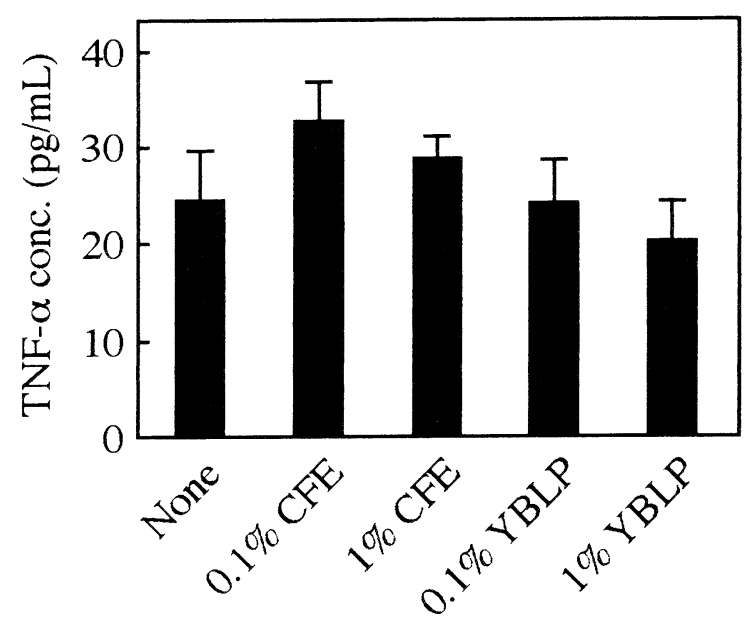

Fig. 1. Effects of dietary CFE and YBLP on production of TNF- $\alpha$ by spleen lymphocytes. Data are the means \pm SE ( $n=4$ or 5 ). There are no significant differences among dietary groups.

that CFE feeding tended to enhance the production of TNF- $\alpha$ by spleen lymphocytes. It has been also reported that some kinds of lactic bacteria belonging to the genus Lactobacillus and Streptocuccus enhance the production of such cytokines as TNF- $\alpha$ in mice and human lymphocytes (18). Therefore, not only cabbage-derived components but also bacteria-related ones or metabolites in CFE may modulate the production of TNF- $\alpha$. However, key components involved in the modulation of TNF- $\alpha$ production have not been identified yet. On the other hand, dietary effects of cabbage products on Ig production remained obscure, but we showed a few years ago that water-soluble components in cabbage stimulated IgA and IgG production of rat MLN lymphocytes in vitro (4). In this study, it was suggested that some components contained in cabbage might stimulate IgA and IgG production of MLN lymphocytes in vivo as well as in vitro. Moreover, CFE feeding increased serum IgG level and IgG productivity of spleen lymphocytes. These results suggest that CFE contributes to the modulation of Ig production in both systemic and intestinal immune systems. 
Table 4. Effects of dietary CFE and YBLP on fatty acids composition of spleen phosphatidylcholine.

\begin{tabular}{|c|c|c|c|c|c|}
\hline & \multirow{2}{*}{ None } & \multicolumn{2}{|c|}{ CFE } & \multicolumn{2}{|c|}{ YBLP } \\
\hline & & $0.1 \%$ & $1 \%$ & $0.1 \%$ & $1 \%$ \\
\hline $14: 0$ & $0.41 \pm 0.02^{\mathrm{a}}$ & $0.32 \pm 0.01^{b}$ & $0.31 \pm 0.03^{b}$ & $0.39 \pm 0.02^{\mathrm{ac}}$ & $0.32 \pm 0.03^{b c}$ \\
\hline $16: 0$ & $34.71 \pm 1.89^{\mathrm{a}}$ & $31.45 \pm 0.27^{\mathrm{ab}}$ & $30.19 \pm 1.35^{b}$ & $35.25 \pm 1.24^{\mathrm{a}}$ & $31.26 \pm 1.05^{\mathrm{ab}}$ \\
\hline $16: 1$ & $0.45 \pm 0.01$ & $0.42 \pm 0.01$ & $0.46 \pm 0.01$ & $0.46 \pm 0.07$ & $0.53 \pm 0.03$ \\
\hline $18: 0$ & $16.42 \pm 0.27$ & $16.74 \pm 0.43$ & $16.40 \pm 0.23$ & $16.28 \pm 0.91$ & $14.88 \pm 1.06$ \\
\hline $18: 1$ & $5.01 \pm 0.09^{\mathrm{ab}}$ & $4.86 \pm 0.03^{b}$ & $5.03 \pm 0.05^{\mathrm{ab}}$ & $5.13 \pm 0.09^{\mathrm{a}}$ & $5.09 \pm 0.13^{\mathrm{ab}}$ \\
\hline $18: 2(n-6)$ & $13.43 \pm 0.38^{\mathrm{ab}}$ & $12.47 \pm 0.15^{\mathrm{ab}}$ & $12.93 \pm 0.21^{\mathrm{ab}}$ & $13.84 \pm 0.83^{\mathrm{a}}$ & $11.93 \pm 0.56^{b}$ \\
\hline $18: 3(n-6)$ & $0.11 \pm 0.04$ & $0.18 \pm 0.01$ & $0.12 \pm 0.02$ & $0.14 \pm 0.03$ & $0.14 \pm 0.03$ \\
\hline $20: 3(n-6)$ & $0.97 \pm 0.04$ & $0.99 \pm 0.02$ & $1.02 \pm 0.04$ & $1.11 \pm 0.18$ & $1.01 \pm 0.06$ \\
\hline $20: 4(n-6)$ & $17.52 \pm 1.32^{\mathrm{ac}}$ & $19.81 \pm 0.18^{\mathrm{bc}}$ & $20.45 \pm 0.74^{b}$ & $16.09 \pm 1.10^{\mathrm{a}}$ & $21.11 \pm 0.45^{\mathrm{b}}$ \\
\hline $22: 6(n-3)$ & $0.27 \pm 0.02^{\mathrm{ac}}$ & $0.31 \pm 0.01^{\mathrm{cd}}$ & $0.34 \pm 0.03^{b d}$ & $0.24 \pm 0.02^{\mathrm{a}}$ & $0.39 \pm 0.02^{b}$ \\
\hline
\end{tabular}

Data are means $\pm \mathrm{SE}(n=5)$, and ${ }^{\text {abcd }}$ values not sharing a common superscript letter are significantly different at $p<0.05$.

YBLP consists purely of young barley leaves, which are rich in dietary fiber accounting for $52.1 \%$ of all nutrients. In the previous study, some water-soluble dietary fibers increased rat serum IgG levels and reinforced IgA productivity of MLN lymphocytes compared to cellulose (1). Similarly, it was reported that indigestible polysaccharides increased IgA level in the small intestine (19). A quite similar tendency was obtained in this study as well. On the other hand, flavonoids predominate in leaves of various cereal crops, and a novel anti-oxidant has been isolated from young green barley leaves $(20,21)$. We have reported that such anti-oxidants as $\alpha$-tocopherol and tea polyphenols modulate Ig or chemical mediator production both in vitro and in vivo (22-25). Therefore, these factors might play a part in the modulation of Ig production by dietary YBLP, but the mechanism has not been revealed yet. In addition, YBLP might serve to prevent obesity because its feeding caused a decrease in food efficiency.

It is considered that the contents of PUFA in immunerelated tissues and cells are an important factor in the modulation of inflammatory response and type I allergy (5-8). We could not observe any effects of CFE or YBLP feeding on fatty acid composition in spleen phospholipids. Therefore, they may have no anti-inflammatory effects. It is necessary to investigate the possibility of deterioration of inflammatory response caused by their feeding.

In conclusion, dietary CFE and YBLP modulate Ig production of rat lymphocytes in both systemic and intestinal immune systems. CFE feeding tends to enhance the production of TNF- $\alpha$ by spleen lymphocytes. It thus seems likely that CFE and YBLP serve as supplemental foodstuffs effective for immune stimulation. It remains to be further investigated whether the modulation of immune parameters by feeding CFE or YBLP is effective in treatment of infectious disease, allergy, cancer and so on.

\section{REFERENCES}

1) Lim BO, Yamada K, Nonaka M, Kuramoto Y, Hung P, Sugano M. 1997. Dietary fibers modulate indices of in- testinal immune function in rats. I Nutr 127: 663667.

2) Yamada K, Tokunaga Y, Ikeda A, Ohkura K, Mamiya S, Kaku S, Sugano M, Tachibana H. 1999. Dietary effect of guar gum and its partially hydrolyzed product on lipid metabolism and immune function of SpragueDawley rats. Biosci Biotechnol Biochem 63: 2163-2167.

3) Kuramoto Y, Yamada K, Tsuruta O, Sugano M. 1996. Effect of natural food colorings on immunoglobulin production in vitro by rat spleen lymphocytes. Biosci Biotechnol Biochem 60: 1712-1713.

4) Kaku S, Yamada K, Hassan N, Watanabe T, Sugano M. 1997. Effect of vegetable extracts on immunoglobulin production by mesenteric lymph node lymphocytes of Sprague-Dawley rats. Biosci Biotechnol Biochem 61: 558-560.

5) Samuelsson B, Dahlen SE, Lindgren JA, Rouzer CA, Serhan CN. 1987. Leukotrienes and lipoxins: structures, biosynthesis, and biological effects. Science 237: 1171-1176.

6) Dahlen SE, Kumlin M, Granstrom E, Hedqvist P. 1986. Leukotrienes and other eicosanoids as mediators of airway obstruction. Respiration 50 (Suppl 2): 22-29.

7) Koga T, Yamato T, Gu JY, Nonaka M, Yamada K, Sugano M. 1994. Diversity in the incorporation into tissue phospholipids and effects on eicosanoid production of trans-monoene fatty acid in rats fed with different dietary proteins. Biosci Biotechnol Biochem 58: 384-387.

8) Yamada K, Mori M, Matsuo N, Shoji K, Ueyama T, Sugano M. 1996. Effect of fatty acids on accumulation and secretion of histamine in RBL-2H3 cells and leukotriene release from peritoneal exudate cells isolated from Wistar rats. J Nutr Sci Vitaminol 42: 301-311.

9) Gu JY, Wakizono Y, Dohi A, Nonaka M, Sugano M, Yamada K. 1998. Effect of dietary fats and sesamin on the lipid metabolism and immune function of SpragueDawley rats. Biosci Biotechnol Biochem 62: 1917-1924.

10) Sugano M, Tsujita A, Yamasaki M, Noguchi M, Yamada K. 1998. Conjugated linoleic acid modulates tissue levels of chemical mediators and immunoglobulins in rats. Lipids 33: 521-527.

11) Lim BO, Yamada K, Sugano M. 1994. Effect of bile acids and lectins on immunoglobulin production in rat mesenteric lymph node lymphocytes. In Vitro Cell Dev 
Biol 30: 407-413.

12) Stepaniak JA, Gould KE, Sun D, Swanborg RH. 1995. A comparative study of experimental autoimmune encephalomyelitis in Lewis and DA rats. J Immunol 155: 2762-2769.

13) Folch J, Lees M, Sloane-Stanley GH. 1957. A simple method for the isolation and purification of total lipides from animal tissues. J Biol Chem 226: 497-509.

14) Claude L, Xavier P. 1987. Thin-layer chromatography of human platelet phospholipids with fatty acid analysis. J Chromatogr 420: 411-416.

15) Duncan DB. 1975. Multiple range tests for correlated and heteroscedastic means. Biometrics 3: 164-176.

16) Bradfield CA, Bjeldanes LF. 1991. Modification of carcinogen metabolism by indolylic autolysis products of Brassica oleraceae. Adv Exp Med Biol 289: 153-163.

17) Beecher CW. 1994. Cancer preventive properties of varieties of Brassica oleracea: a review. Am J Clin Nutr 59 (5 Suppl): 1166S-1170S.

18) Pereyra BS, Aattouri N, Lemonnier D. 1997. Role of food in the stimulation of cytokine production. Am J Clin Nutr 66: 521S-525S.

19) Kudoh K, Shimizu J, Wada M, Takita T, Kanke Y, Innami S. 1998. Effect of indigestible saccharides on B lymphocyte response of intestinal mucosa and cecal fermentation in rats. J Nutr Sci Vitaminol 44: 103-112.

20) Kitta K, Hagiwara Y, Shibamoto T. 1992. Antioxidative activity of an isoflavonoid, 2"-o-glycosylisovitexin isolated from green barley leaves. J Agric Food Chem 40: 1843-1845.

21) Osawa T, Katsuzaki H, Hagiwara Y, Hagiwara H, Shibamoto T. 1992. A novel antioxidant isolated from young green barley leaves. I Agric Food Chem 40: 1135-1138.

22) Gu JY, Nonaka M, Yamada K, Yoshimura K, Takasugi M, Ito Y, Sugano M. 1994. Effect of sesamin and $\alpha$ tocopherol on the production of chemical mediators and immunoglobulins in Brown-Norway rats. Biosci Biotechnol Biochem 58: 1855-1858.

23) Hung P, Yamada K, Lim BO, Mori M, Yuki T, Sugano M. 1997. Effect of unsaturated fatty acids and $\alpha$-tocopherol on immunoglobulin levels in culture medium of rat mesenteric lymph node and spleen lymphocytes. $J$ Biochem 121: 1054-1060.

24) Matsuo N, Yamada K, Yamashita K, Shoji K, Mori M, Sugano M. 1996. Inhibitory effect of tea polyphenols on histamine and leukotriene B4 release from rat peritoneal exudate cells. In Vitro Cell Dev Biol 32: 340-344.

25) Yamada K, Hung P, Yoshimura K, Taniguchi S, Lim BO, Sugano M. 1996. Effect of unsaturated fatty acids and antioxidants on immunoglobulin production by mesenteric lymph node lymphocytes of Sprague-Dawley rats. J Biochem 120: 138-144. 